\title{
Biventricular Pacing Optimization by Means of the Dyssynchrony Parameter
}

\author{
Pavel Jurak ${ }^{1,2}$, Pavel Leinveber ${ }^{2}$, Josef Halamek ${ }^{1}$, Filip Plesinger ${ }^{1}$, Tereza Postranecka ${ }^{2}$, \\ Jolana Lipoldova ${ }^{2}$, Miroslav Novak ${ }^{2}$ \\ ${ }^{1}$ Institute of Scientific Instruments of the Czech Academy of Sciences, Brno, Czech Republic \\ ${ }^{2}$ International Clinical Research Center, St. Anne’s University Hospital, Brno, Czech Republic
}

\begin{abstract}
To improve Cardiac Resynchronization Therapy (CRT), different interventricular delay (VVD) settings can be used. However, relatively small VVD induced hemodynamic changes cannot be measured by standard echocardiographic methods. The QRS complex duration $(Q R S d)$ is mostly the main criterion. Here we introduce a new dyssynchrony parameter (DYS) that is able to more accurately detect improved electrical synchrony.

Methods: 12-lead $5 \mathrm{kHz}$ ECG during 3-10 minute rest period was measured in 46 patients with CRT OFF and CRT ON with VVD 0 ms (CRT_0) and -20 ms (CRT_20). We detected QRSd and the dyssynchrony parameter DYS as the time difference between 500-1000 Hz averaged envelopes positions in the V1 and V6 leads in the QRS complex region.

Results: 32 of 46 patients had a positive CRT response manifested by QRSd shortening and a DYS decrease. 28 of 32 patients had a positive $L V$ pre-excitation effect: additional QRSd shortening of $4.7 \pm 5.9 \mathrm{~ms}$ and a DYS decrease of $12.6 \pm 7.5 \mathrm{~ms}$. The correlation coefficient of QRSd and DYS changes (CRT_0 vs CRT_20) was 0.23 and indicates information diversity. The DYS parameter differs from QRSd and provides a significantly higher response to $V V$ delay changes $(p<0.001)$.
\end{abstract}

\section{Introduction}

Cardiac resynchronization therapy (CRT) provides major protection against heart failure events in patients with left bundle branch block (LBBB). While normal left ventricular (LV) contraction is rapid and synchronous, LBBB exhibits significant regional contraction delay: the septal region is activated first and contracts against a nonactivated lateral LV wall [1]. Thus, the lateral wall region is being stretched and, when finally activated, contracts against a high preload. This mechanical dyssynchrony impairs the efficiency of ventricular contraction, reduces stroke volume, and increases workload and LV volumes. Optimising interventricular stimulation delay (VVD) in patients with CRT can be associated with additionally decreased dyssynchrony, improved cardiac output and finally in a reduction of heart failure symptoms [2]. However, while atrioventricular delay change (AVD) has a strong influence on blood filling and can be detected by echocardiographic methods, VVD causes mostly small hemodynamic changes very often under the echocardiographic measurement error threshold. Consequently, the main criterion of VVD positive effect on ventricular dyssynchrony is inaccurate QRS duration (QRSd) shortening detection.

In this study, we introduce a new dyssynchrony parameter (DYS) that is computed from ultra-high frequency QRS components. The ultra-high-frequency nature can be found in the rapid depolarization phase 1 of contractile cell action potentials. Electrical depolarization of the myocardium represents the initiation of tension in contractile cells and subsequent mechanical activity.

\section{Methods}

We used an ultra-high-frequency high-dynamic-range ECG technique. Pilot results and the DYS parameter were introduced on set of CRT patients in [3,4].

\subsection{Data recording, protocol and subjects}

A 12-lead ECG was collected at $5 \mathrm{kHz}$ with a dynamic range of 26 bits and a $2 \mathrm{kHz}$ pass band (M\&I Prague, CZ) - UHF-ECG. Measurements were taken at the International Clinical Research Center at St. Anne's University Hospital, Brno, Czech Republic.

We measured and analysed 46 left LBBB patients referred for CRT. Age mean $/ \mathrm{max} / \mathrm{min}$ 67/80/35, male/female $37 / 9$, LBBB pattern $70 \%$. The UHF-ECG data were collected over 3-10 minutes in the resting supine position with CRT OFF and CRT ON with interventricular delay VVD 0 ms (CRT_0) and $-20 \mathrm{~ms}$ (CRT_20). UHF Solver (ISI AVCR, Brno, CZ) software developed specifically for UHF-ECG data processing was used. 

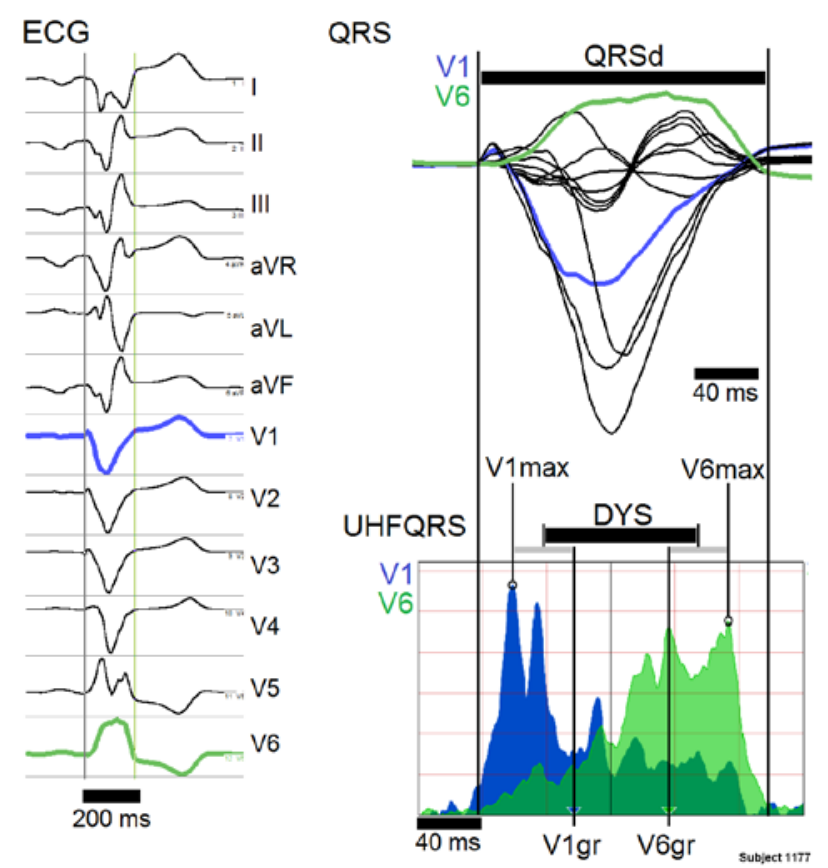

Figure 1. LBBB patient. Left panel: averaged 12-lead ECG, Right panel, upper part: averaged QRS complexes with manual identification of QRS onset and QRS end. Leads V1 (green) and V6 (blue). Right panel, bottom part: averaged 500-1000 Hz V1 and V6 envelopes UHFQRS, blue represents UHFQRS distribution in the septum and right ventricle (RV) and green represents UHFQRS distribution in the LV lateral wall. The mutual shift between green and blue distribution shows dyssynchrony.

QRSd: the width of the QRS complex (181 ms), DYS: dyssynchrony parameter computed from maximums V1max, V6max and centre of gravity V1gr, V6gr (99 $\mathrm{ms})$.

\subsection{Processing}

Stimulation peaks were detected and removed during CRT ON. Figure 3 left panel during CRT ON shows stimulation peaks and QRS complexes after its removal. QRS complexes were detected and sorted into categories using a robust multichannel approach [Plesinger 2014]. This technique was used to distinguish a regular rhythm from abnormal QRS shapes to focus the analysis primarily on the sinus (dominant) rhythm. The amplitude envelopes were computed in a frequency band of 500$1000 \mathrm{~Hz}$ by the Hilbert transform. Amplitude envelopes were averaged with an R-wave trigger and smoothened in low-pass $0-40 \mathrm{~Hz}$ - UHFQRS (Figure 1).

From UHFQRS we detected: the position of maximums V1max and V6max and the position of gravity V1gr and V6gr - Figure 1. We computed the DYS parameter as time distance between the centre of V1maxV1gr and the centre of V6max and V6gr. This is a compromise between the DYS estimated only from maximums suffering from great variability and DYS estimated from the centres of gravity, which is undervalued. The QRSd parameter was manually detected from all 12 averaged leads - Figure 1,3.
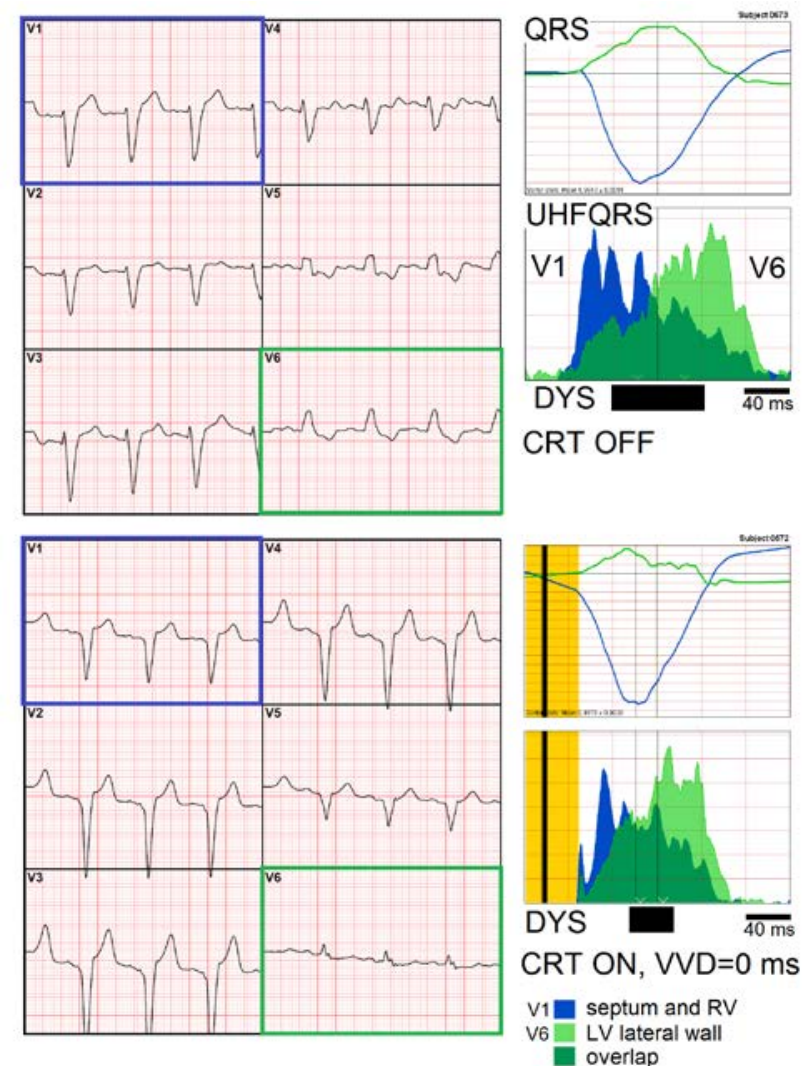

Figure 2. The effect of biventricular pacing. Left panel: V1-V6 ECG signal, right panel: average V1 and V6 QRS complexes and UHFQRS.

Upper panel: CRT OFF, QRSd $180 \mathrm{~ms}$, DYS $104 \mathrm{~ms}$ bottom panel: CRT ON, VVD 0 ms, QRSd 139 ms, DYS $52 \mathrm{~ms}$. ECG with eliminated stimulation peaks, the yellow vertical bar in UHFQRS defines the region with stimulus peak elimination. The black vertical bar defines stimulus peak positions.

\section{Results}

The results of QRSd and DYS during CRT OFF, CRT_0 and CRT_20 are demonstrated in the tables. The significance of the differences was tested using a paired T-test. Table 1 includes the results of all 46 CRT patients. The QRSd and DYS parameters reduce significantly during CRT_0 in comparison with CRT_OFF. While there are no significant differences in the QRSd parameter during CRT_0 vs CRT_20, the DYS shows a significant reduction $(\mathrm{p}<0.0001)$. Table 2 includes 32 patients $(70 \%)$ with a positive CRT response. Positivity was assessed by 
QRSd shortening during CRT_0 of $5 \mathrm{~ms}$ and higher. Differences in QRSd and DYS between CTR_OFF and CRT_0 were significant $(\mathrm{p}<0.00001)$. Differences between different VVD settings were significant both in QRSd $(\mathrm{p}<0.001)$ and DYS $(\mathrm{p}<0.0001)$. Table 3 represents 14 patients with a negative CRT response. Only minor and overall insignificant differences of QRSd and DYS parameters were observed between CRT OFF and ON.

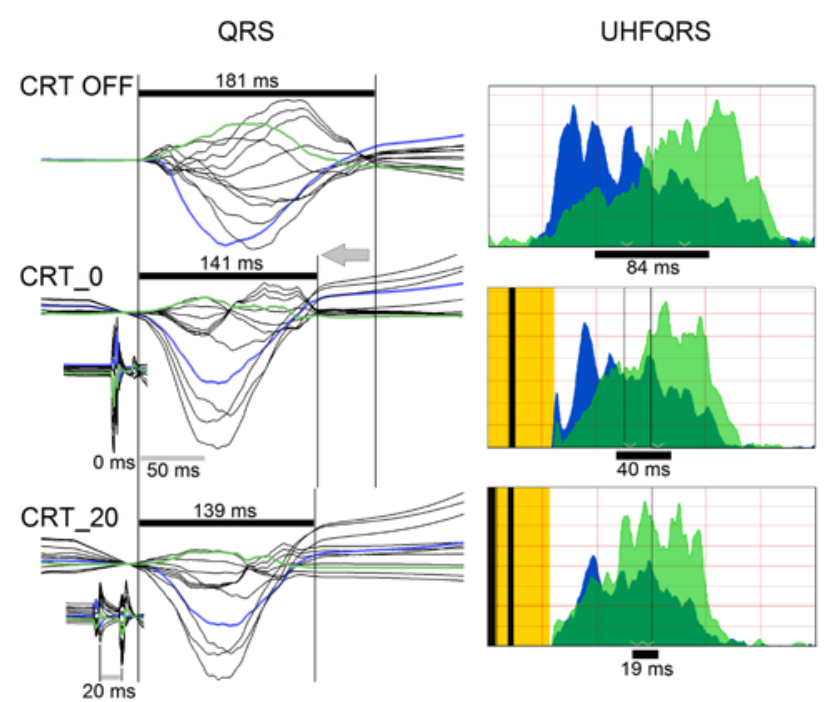

Figure 3. The effect of VVD optimization on QRS duration and UHFQRS distribution. Left panel: averaged QRS complexes, right panel: averaged UHFQRS, from the top: without biventricular pacing (CRT OFF), with biventricular pacing and VVD zero ms (CRT_0), with biventricular pacing and $20 \mathrm{~ms}$ pre-excitation of the $\mathrm{LV}$ lateral wall - VVD minus $20 \mathrm{~ms}$ (CRT_20). There is a strong QRSd decrease in CRT_0 (40ms), but a minimal QRSd decrease during pre-excitation (2 ms). The UHFQRS morphology clearly identify a green shift to the left - the synchronization effect of pre-excitation.

\section{Discussion}

The main goal of CRT biventricular pacing optimization is to meet the individual patient's needs by determining the optimal time setting of AVD and consequently VVD. An increased hemodynamic outcome and a long-term positive follow up are the basic and crucial criteria. However, large multicentre studies are needed to verify these criteria due to the low sensitivity and specificity of the evaluated parameters and the patient's subjective questionnaire responses. On the other hand, measuring cardiac electrical activity is a deterministic and highly reproducible approach with a minimal influence of subjective assessment.

In this study, we compared two outputs of cardiac electrical activity - commonly used QRSd and new information separated from the UHFQRS - DYS parameter. We demonstrated the different reactions of QRSd and DYS on two VVD settings in Tables 1-3. 32 of 46 patients had a positive CRT response manifested by QRSd shortening and a DYS decrease. 28 of 32 patients had a positive LV pre-excitation effect: an additional QRSd shortening of $4.7 \pm 5.9 \mathrm{~ms}$ and a DYS decrease of $12.6 \pm 7.5 \mathrm{~ms}$. The correlation coefficient of QRSd and DYS changes (CRT_0 vs CRT_20) was 0.23 and indicates information diversity. The DYS parameter differs from QRSd and provides a significantly higher response to VVD changes ( $p<0.001$ ). The results demonstrate more significant differences between positive and negative reactions in the DYS parameter.

Table 1. All 46 CRT patients, QRSd and DYS mean and SD in milliseconds, ${ }^{*} \mathrm{p}<0.01, \quad{ }^{* *} \mathrm{p}<0.001$, $* * * \mathrm{p}<0.0001, * * * * \mathrm{p}<0.00001$

\begin{tabular}{lcc}
\hline & QRSd & DYS \\
\hline CRT OFF & $158 \pm 25$ & $65 \pm 36$ \\
& $* * * *$ & $* * * *$ \\
CRT_0 & $133 \pm 18$ & $26 \pm 19$ \\
& NS & $* * *$ \\
CRT_20 & $132 \pm 18$ & $18 \pm 19$ \\
\hline
\end{tabular}

Table 2. 32 patients with a positive CRT_0 response

\begin{tabular}{lcc}
\hline & QRSd & DYS \\
\hline CRT OFF & $165 \pm 17$ & $83 \pm 27$ \\
& $* * * *$ & $* * * *$ \\
CRT_0 & $132 \pm 15$ & $31 \pm 19$ \\
& $* *$ & $* * * *$ \\
CRT_20 & $127 \pm 16$ & $20 \pm 21$ \\
\hline
\end{tabular}

Table 3. 14 patients with a negative CRT_0 response

\begin{tabular}{lcc}
\hline & QRSd & DYS \\
\hline CRT OFF & $141 \pm 31$ & $25 \pm 27$ \\
& NS & NS \\
CRT_0 & $136 \pm 24$ & $16 \pm 14$ \\
& NS & NS \\
CRT_20 & $142 \pm 21$ & $15 \pm 16$ \\
\hline
\end{tabular}

The illustrative examples in Figure 4 clarify the graphical properties of UHFQRS component distribution in V1 and V6 leads. The DYS parameter represents a clearly interpretable numerical parameter. Nevertheless, the graphical inspection is irreplaceable and offers important information about depolarization distribution over time and in electrodes. This information is especially crucial for individual CRT optimization.

The simple and schematic interpretation of UHFQRS is demonstrated in Figure 5. Time shifting of the green triangle to the left represents the electrical synchronization of the RV and LV. Depending on the degree of blue and green overlap, a greater or lesser 
degree of electrical synchronization can be considered. This technique can be used both during CRT candidate selection and following CRT parameter optimization.

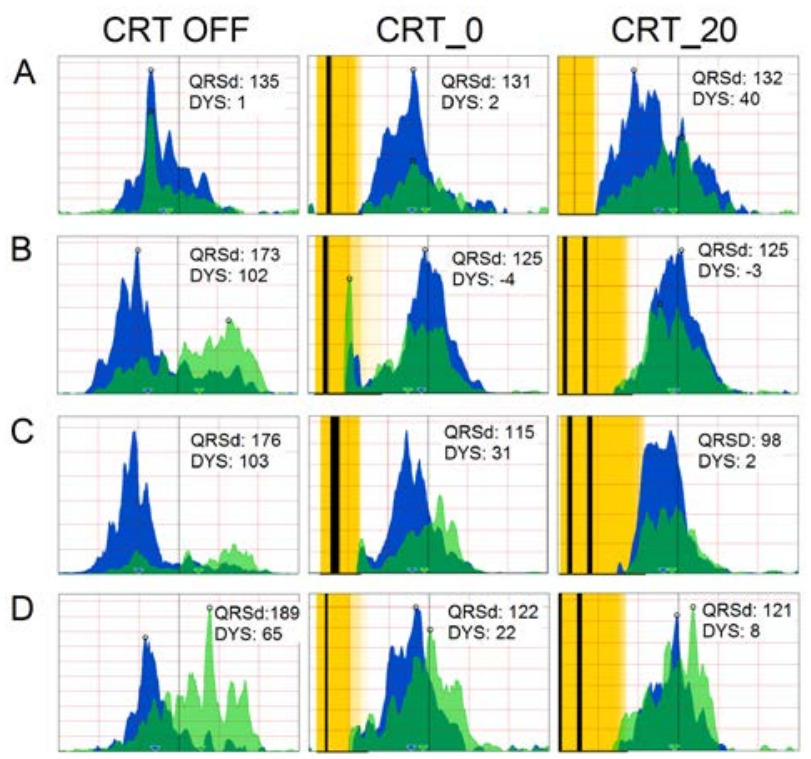

Figure 4. Examples of different resynchronization effects on electrical depolarization distribution.

A: subject with low dyssynchrony before CRT ( $<5 \mathrm{~ms})$, CRT_0 has a minimal resynchronization effect $(<5 \mathrm{~ms})$, CRT_20 even worsens the dyssynchrony (40 ms). B-D: subjects with large dyssynchrony before CRT (102/103/65 ms), B: CRT_0 reduces dyssynchrony to $<5$ $\mathrm{ms}$, but there is no longer a positive effect of CRT_20 $(<5$ $\mathrm{ms})$.

C: CRT_0 reduces dyssynchrony to $31 \mathrm{~ms}$ and CRT_20 causes an additional improvement to $<5 \mathrm{~ms}$.

D: CRT_0 reduces dyssynchrony to $22 \mathrm{~ms}$ and CRT_20 causes an additional improvement to $<10 \mathrm{~ms}$.

In [3] we firstly introduced the UHFQRS technique to detect ventricular dyssynchrony. We mentioned that the main limitation of UHFQRS is a lack of complementary technology that is able to measure the ventricular mechanical properties and hemodynamic improvement at a comparable time resolution and accuracy [6]. This greatly complicates direct verification by standard and respected methods such as echocardiography or MRI. The QRS duration parameter is not generally recognized as sufficient proof of the positive effect of CTR optimization.
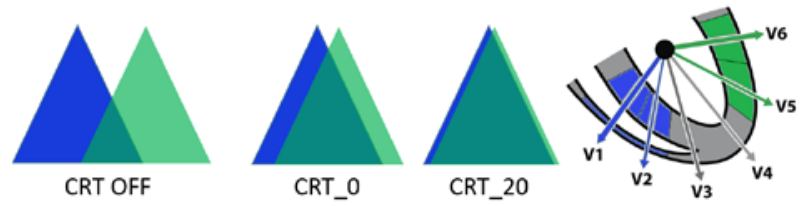

Figure 5. A schematic interpretation of UHFQRS electrical dyssynchrony eliminated by CRT.
Technical aspects: we demonstrated UHFQRS in a 5 $\mathrm{kHz}$ data and frequency range of $500-1000 \mathrm{~Hz}$. A conventional $1 \mathrm{kHz}$ ECG and a frequency range of 150$400 \mathrm{~Hz}$ seems to be sufficient to obtain basic information about electrical dyssynchrony and DYS parameter computation.

\section{Conclusion}

The DYS parameter estimated from UHFQRS provides new information about ventricular dyssynchrony and can potentially be used for CRT VVD setting optimization.

\section{Acknowledgements}

The research was supported by project no. P102/12/2034 from the Grant Agency of the Czech Republic and by MEYS CR (LO1212), its infrastructure by MEYS CR and EC (CZ.1.05/2.1.00/01.0017) and by ASCR (RVO:68081731); European Regional Development Fund - Project FNUSA-ICRC CZ.1.05/1.1.00/02.0123.

\section{References}

[1] Ott P. Cardiac Resynchronization Therapy: A New Therapy for Advanced Congestive Heart Failure. Am J Geriatr Cardiol. 2005;14(1):31-34

[2] Naqvi TZ. Echocardiography-Guided Biventricular Pacemaker Optimization. JACC CI. 2010;3(11):1168-1180

[3] Jurak P, Halamek J, Plesinger F, et al. An additional marker of ventricular dyssynchrony. In 2015 Computing in Cardiology (CinC). ISSN 2325-8861, Sept 2015; 77-80.

[4] Reichlova T, Jurak P, Halamek J, et al. Cardiac Resynchronization Efficiency Estimation by New UltraHigh-Frequency ECG Dyssynchrony Descriptor Measurement. Computing in Cardiology (CinC) 2015.

[5] Plesinger F, Jurco J, Jurak P, Halamek J. Robust multichannel QRS detection. Computing in Cardiology (CinC) 2014. 557-560. ISBN: 978-1-4799-4346-3.

[6] Leinveber P. Halamek J, Jurak P, Meluzin J. The relationship between mechanical and electrical dyssynchrony. Computing in Cardiology (CinC) 2016.

Address for correspondence.

Pavel Jurak

Institute of Scientific Instruments of the CAS,

Kralovopolska 147, 61264 Brno, Czech Republic

E-mail: jurak@isibrno.cz 